\title{
Nanoscale
}

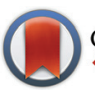

CrossMark \&click for updates

Cite this: Nanoscale, 2016, 8, 9272

Received 20th January 2016, Accepted 11th April 2016

DOI: 10.1039/c6nr00529b

www.rsc.org/nanoscale

\section{Charge-tunnelling and self-trapping: common origins for blinking, grey-state emission and photoluminescence enhancement in semiconductor quantum dots $\uparrow$}

\begin{abstract}
M. A. Osborne* and A. A. E. Fisher
Understanding instabilities in the photoluminescence (PL) from light emitting materials is crucial to optimizing their performance for different applications. Semiconductor quantum dots (QDs) offer bright, size tunable emission, properties that are now being exploited in a broad range of developing technologies from displays and solar cells to biomaging and optical storage. However, instabilities such as photoluminescence intermittency, enhancement and bleaching of emission in these materials can be detrimental to their utility. Here, we report dielectric dependent blinking, intensity-"spikes" and low-level, "grey"state emission, as well as PL enhancement in ZnS capped CdSe QDs; observations that we found consistent with a charge-tunnelling and self-trapping (CTST) description of exciton-dynamics on the QD-host system. In particular, modulation of PL in grey-states and PL enhancement are found to have a common origin in the equilibrium between exciton charge carrier core and surface-states within the CTST framework. Parameterized in terms of size and electrostatic properties of the QD and its nanoenvironment, the CTST offers predictive insight into exciton-dynamics in these nanomaterials.
\end{abstract}

\section{Introduction}

Semiconductor quantum dots (QDs) are now widely used as light harvesters and emitters across a spectrum of applications, from sensing to photovoltaics and display technology. ${ }^{1}$ Many of their optical properties, including size tuneable emission, can be described by simple particle-in-a-box quantum mechanics and electrostatics. ${ }^{2}$ Photoluminescence intermittency (PI),$^{3-6}$ enhancement $(\mathrm{PE})^{7-10}$ and bluing, ${ }^{1-14}$ charging, ${ }^{15}$ multi-level emission ${ }^{16}$ and "grey"-states ${ }^{17,18}$ are additional properties that are generally detrimental to QD performance, but are not well understood.

While several models have been successful in describing the characteristic statistics of $\mathrm{PI},{ }^{19-24}$ many remain untested against the complete set of experimentally observed dependencies, including excitation wavelength ${ }^{25,26}$ and intensity, ${ }^{27,28}$ QD capping $^{29}$ and the dielectric constant of the QD nanoenvironment. ${ }^{30,31}$ Moreover, descriptions of the grey-state and PE are

Department of Chemistry, School of Life Sciences, University of Sussex, Falmer, Brighton, BN1 9QJ, UK. E-mail: m.osborne@sussex.ac.uk

$\dagger$ Electronic supplementary information (ESI) available: Additional notes, equations, figures and tables defining kinetic, energetic and geometric parameters used in simulations. Additional experimental data showing TPL cut-off times, grey-states in CdSe/CdS core-shell QDs and irreversible PL enhancement. See DOI: $10.1039 / \mathrm{c} 6 \mathrm{nr} 00529 \mathrm{~b}$ few and largely qualitative in nature. That said, charge-tunnelling and diffusion-controlled electron-transfer (DCET) ${ }^{24}$ models of PI have been successfully extended to account for dielectric dependent blinking ${ }^{30}$ and the recently observed role of the biexciton in PI. ${ }^{32}$ Blinking in QDs is commonly characterized by a truncated power-law (TPL), $P(t)=A t^{-\alpha} e^{-t / \tau_{\mathrm{c}}}$ that describes the probability density distributions (PDDs) of switching times between bright (on) and dark (off) states of the QD. Importantly, while the exponent, $\alpha$ and truncation time, $\tau_{\mathrm{c}}$, have explicit origins in the DCET model proposed by Tang and Marcus, ${ }^{21}$ the relationship between these parameters and measurable properties of the QD and its nanoenvironment is less overt. On the other hand, charge-tunnelling models developed by Verberk et $a .^{20}$ and separately Kuno et $a .^{33}$ define $\alpha$ explicitly in terms of QD confinement and trap potentials. Isaac et al. ${ }^{30}$ further showed a correlation between $\alpha$ and the dielectric constant of the QD surround that could be accounted for, albeit qualitatively, by relating the trap potential to the dielectric dependent reaction-field of the QD-host.

Many experiments have shown the PL intensity trajectory of single QDs is richer in quantum yield (QY) variation than can be described by PI alone. For example, electrochemically controlled blinking studies have revealed two types of PI; (A-type) conventional blinking, associated with the Auger quenching of band-edge emission in charged-QDs and (B-type) blinking where PL is suppressed by a fast, non-radiative exciton-recom- 
bination pathway that intercepts normal internal conversion of the exciton to the band-edge. ${ }^{34}$ Furthermore, PE (and PL decay) and long-lived grey-states add complexity to the envelope of PL instabilities observed in QDs. Studies of PE and blinking suppression have shown the critical role played by charge-induced electric fields ${ }^{35,36}$ and adsorbates ${ }^{37,38}$ in the activation and passivation of QD surface-states associated with reversible changes in QY, while irreversible changes have been attributed to the structural annealing ${ }^{39,40}$ and photoinduced oxidation of the QD. ${ }^{41,42}$ In particular, the influence of surface holetrapping on PL modulation in QDs was recognized through early investigations of PI suppression by electron-donating organic ligands. $^{43}$ More recent experiments, in which surface-trap numbers have been closely-controlled at the QD-substrate interface, have highlighted hole-trapping as central to the description of the anomalous blinking kinetics observed in these engineered systems. ${ }^{44}$ Despite a plethora of data and numerous descriptions of PL dynamics in QDs, a comprehensive understanding of the interplay between PI, grey-states and PE remains elusive.

Here, we report experimental evidence for dielectric and size dependent PI statistics and the observation of charge dependent "spikes" and "grey"-states, as well as irreversible PE in the PL intensity trajectory of individual QDs. The results are found consistent with; (i) a charge-tunnelling and self-trapping (CTST) description of the exciton charge-carrier dynamics in the QD-host; (ii) a biexciton mechanism for charge switching between QD surface and core-states and; (iii) a simple atomistic model of QD degradation that leads naturally to QY enhancement in the QD. Parameterised in terms of macroscopic properties of the QD and the support medium, the model we advance, reproduces PI statistics, grey-state emission intensities and the temporal envelope of PL enhancement and decay observed in our experiments with good quantitative accuracy. Importantly the findings support a common origin for blinking, the grey-state and PE through differences in the exciton-hole dynamics between the neutral-state and core and surface-charged ionised-states of the QD. The control of PL from QDs and other nanomaterials is critical to their performance in applications; whether it be suppressed PI for brighter QDs in displays, ${ }^{45}$ enhanced PI for better localisation in superresolution imaging ${ }^{46}$ or engineered $\mathrm{PE}$ for $\operatorname{sensing}^{47}$ and optical storage. ${ }^{48}$ The CTST description of QD photodynamics advanced here provides a physically insightful and predictive basis for tailoring QD properties for specific applications.

\section{Results and discussion}

\section{Dielectric dependent QD blinking}

PL intensity trajectories of single core-shell, CdSe-ZnS QDS (Lumidot 590, Sigma-Aldrich, UK) were recorded in five supporting media of different dielectric constants, $p$-terphenyl $\left(\mathrm{pT}, \varepsilon_{\mathrm{m}}=2.12\right)$, polystyrene (PS, $\left.\varepsilon_{\mathrm{m}}=2.53\right)$, glass $\left(\mathrm{SiO}_{x}, \varepsilon_{\mathrm{m}}=\right.$ $3.8)$, poly( $N$-vinylpyrrolidone) (PVP, $\varepsilon_{\mathrm{m}}=4.8$ ) and poly-vinylalcohol (PVA, $\left.\varepsilon_{\mathrm{m}}=14\right)$. Qualitatively, trajectories were observed to shift from largely PL-on to mostly PL-off with increasing host dielectric constant, $\varepsilon_{\mathrm{m}}$ (Fig. 1a), a feature which is manifest quantitatively in the least-squares fitting of the TPL to log$\log$ PDDs of on- and off-switching times (Fig. 1b). ${ }^{5,21,32}$ In these PDDs, off-times decay more rapidly for QDs in media of low dielectric constant ( $\mathrm{pT}, \alpha_{\mathrm{off}}=1.75 \pm 0.01, \varepsilon_{\mathrm{m}}=2.12$ ) compared to those in more polarisable nanoenvironments (PVA, $\alpha_{\text {off }}=1.45 \pm 0.01, \varepsilon_{\mathrm{m}}=14$ ), while on-time PDDs were seen to truncate at earlier times with increasing $\varepsilon_{\mathrm{m}}$ (Fig. 1b). The systematic change in $\alpha_{\text {off }}$ and $\alpha_{\text {on }}$ with $\varepsilon_{\mathrm{m}}$ is evident from the histograms of power law exponents extracted from fitting the TPL to individual QD on/off-time PDDs (Fig. 1c and 1d). While onoff exponents display normal-like distributions for all dielectric media, reflecting heterogeneity in the host and QD sizes, mean values of $\alpha_{\text {on/off }}$ showed a systematic decrease with increasing $\varepsilon_{\mathrm{m}}$ (Table $\mathrm{S} 1$ in ESI $\dagger$ ). In contrast, the distributions of exponential cut-off times, $\tau_{\mathrm{c}}$ (on/off) were found generally more uniform, with average off-time truncations appearing constant $\left(\tau_{\mathrm{c}}(\mathrm{off}) \sim 100 \pm 30 \mathrm{~s}\right)$ across the dielectric hosts, at least within the large errors that are associated with the low sampling of events at long-times. Distributions of $\tau_{\mathrm{c}}$ (on), however, tend toward log-normal with most probable values exhibiting an inverse dependence on $\varepsilon_{\mathrm{m}}$ (Fig. S1 in ESI $\dagger$ ). We show below that within the CTST description of PI, the TPL exponents and cut-off times are strongly correlated with the stabilisation of exciton charge-carriers on the host-matrix and QD-host interface.

\section{Charge-tunnelling kinetics}

The CTST model is based on a five-state system (Fig. 2a) with QD photodynamics described by charge-tunnelling between: the neutral, emissive core-exciton state $\left(\mathrm{X}_{00}\right)$; the "dark", corecharged $\left(\mathrm{X}_{10}{ }^{+}\right)$and "bright", surface-charged $\left(\mathrm{X}_{01}{ }^{+}\right)$ionised exciton-states; a transient exciton-hole, surface-state $\left(\mathrm{X}_{00}{ }^{\mathrm{h}}\right)$ in equilibrium with the core-exciton. Exciton-hole surface-states $\left(\mathrm{X}_{10}{ }^{+\mathrm{h}}\right.$ and $\left.\mathrm{X}_{01}{ }^{+\mathrm{h}}\right)$ also persist in the ionised QD under excitation, albeit in different charge-dependent equilibria with their respective core-exciton states $\left(\mathrm{X}_{10}{ }^{+}\right.$and $\left.\mathrm{X}_{01}{ }^{+}\right)$. The probability of finding the QD in a particular state is governed by a set of state-filling rate equations with excitation, radiative and non-radiative relaxation constants, $k_{\mathrm{x}}, k_{\mathrm{r}}$ and $k_{\mathrm{nr}}$ and chargecarrier tunnelling constants ${k_{\mathrm{i}}}^{+},{k_{\mathrm{i}}}^{-},{k_{\mathrm{h}}}^{+}$and $k_{\mathrm{h}}{ }^{-}$, where $(+)$and $(-)$ indicate forward and back-tunnelling processes, to and from the ionised-state (i) or exciton-hole (h) surface-state. The kinetic scheme is evolved in time using standard stochastic simulation methods, with steady-state approximations applied where appropriate (note S1 and eqn (S1) in ESI $\dagger$ ).

In the PL emission cycle the QD is excited from the groundstate to $\mathrm{X}_{00}\left(k_{\mathrm{x}} \sim 10^{5} \mathrm{~s}^{-1}\right)$, where the exciton-hole rapidly establishes an equilibrium, $\mathrm{X}_{00} \leftrightarrow \mathrm{X}_{00}^{\mathrm{h}}$, with the exciton-hole surface state via charge-carrier tunnelling $\left(k_{\mathrm{h}}{ }^{ \pm} \sim 10^{13} \mathrm{~s}^{-1}\right)$. Typically, relaxation from $\mathrm{X}_{00}$ is dominated by radiative recombination $\left(k_{\mathrm{r}} \sim 10^{7} \mathrm{~s}^{-1}\right)$ with $\mathrm{X}_{00}{ }^{\mathrm{h}}$-state sampling acting to modulate QD emission. Slower tunnelling of the exciton-electron to traps in the QD support medium $\left(k_{\mathrm{i}}^{+} \sim 10^{4}-10^{-1} \mathrm{~s}^{-1}\right)$ renders the QD charged and a probability $p$ is placed on finding the nanocrystal in either the core $\mathrm{X}_{10}{ }^{+}$or surface $\mathrm{X}_{01}{ }^{+}$charged ionised- 
(a)
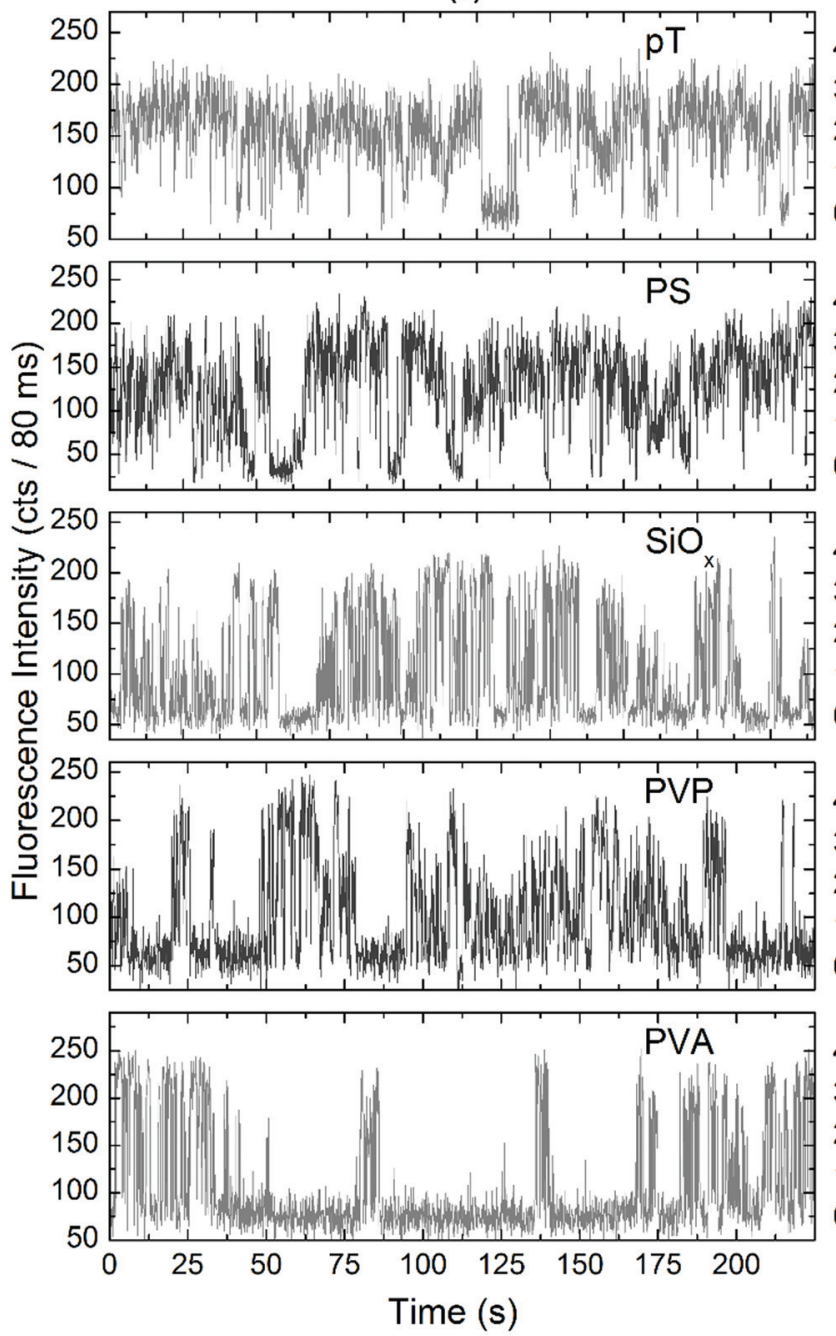

(b)
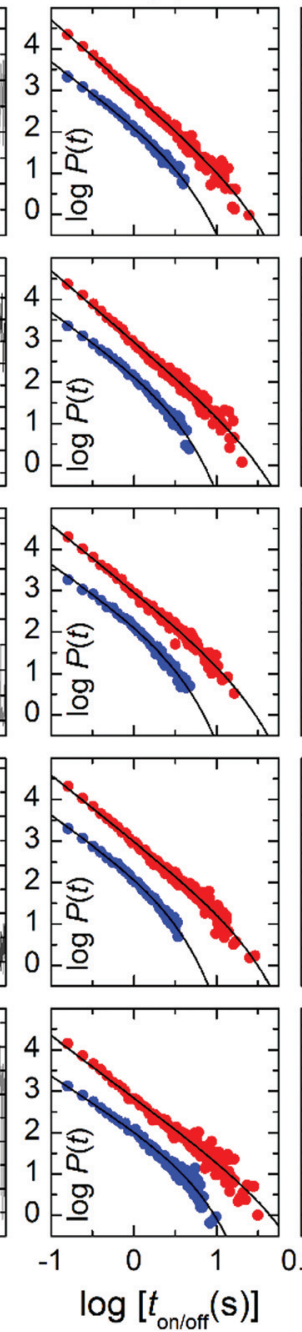

(c)
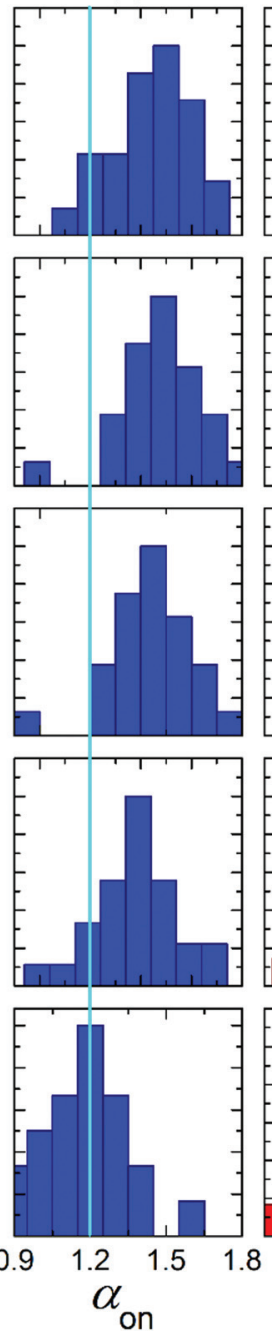

(d)
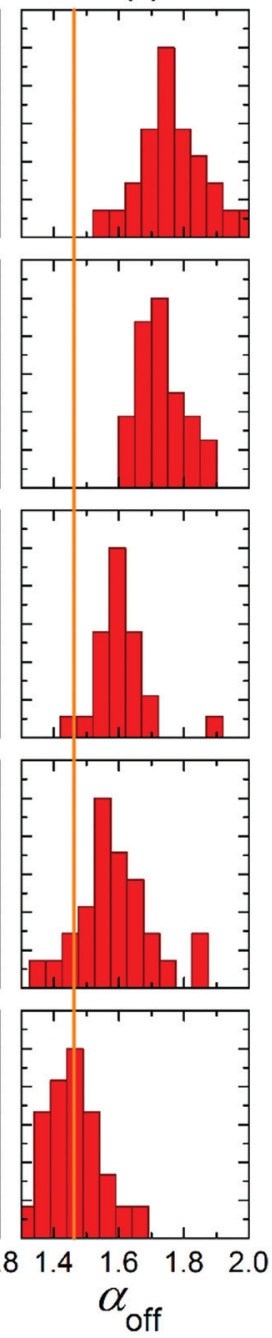

Fig. 1 Dielectric dependent blinking. (a) Extracts from experimental PL intensity trajectories for CdSe-ZnS QDs (Lumidot 590) in pT ( $\varepsilon_{\mathrm{m}}=2.12$ ), PS $\left(\varepsilon_{\mathrm{m}}=2.53\right), \mathrm{SiO}_{x}\left(\varepsilon_{\mathrm{m}}=3.8\right), \operatorname{PVP}\left(\varepsilon_{\mathrm{m}}=4.8\right)$ and PVA $\left(\varepsilon_{\mathrm{m}}=14\right)$. (b) Log-log PDDs for on- and off-times with fits (black lines) of the truncated powerlaw (TPL) function, $P(t)=A t^{-\alpha} e^{-t / \tau_{c}}$. Data points represent averages of more than 20000 on-off events from over 25 QDs per dielectric support. (c) and (d) Histograms of $\alpha_{\text {on }}$ and $\alpha_{\text {off }}$ exponents recovered from TPL fits. Lines through the mean $\alpha_{\text {on }}$ (cyan) and $\alpha_{\text {off }}$ (orange) for PVA highlight the trend to higher exponents with decreasing $\varepsilon_{\mathrm{m}}$.

states. Non-radiative, Auger quenching $\left(k_{\mathrm{nr}} \sim 10^{9} \mathrm{~s}^{-1}\right)$ of the trion dominates $\mathrm{X}_{10}{ }^{+}$relaxation, while radiative recombination resumes in the neutral-core, $\mathrm{X}_{01}{ }^{+}$-state. ${ }^{19,20,33}$ Ultimately, the ionised-states are neutralised by slow back-tunnelling of the host-trapped electron $\left(k_{\mathrm{i}}^{-} \sim 10^{3}-10^{-3} \mathrm{~s}^{-1}\right)$. Thus, blinking arises from the stochastic switching processes $\mathrm{X}_{00} \leftrightarrow \mathrm{X}_{10}{ }^{+}$and $\mathrm{X}_{01}{ }^{+} \leftrightarrow \mathrm{X}_{10}{ }^{+}$, while the PL intensity of the emissive states, $\mathrm{X}_{00}$ and $\mathrm{X}_{01}{ }^{+}$, are also modulated by the equilibrium constant, $k_{\mathrm{h}}{ }^{+} / k_{\mathrm{h}}{ }^{-}$, that controls the exciton-hole surface sampling processes, $\mathrm{X}_{00} \leftrightarrow \mathrm{X}_{00}{ }^{\mathrm{h}}$ and $\mathrm{X}_{01}{ }^{+} \leftrightarrow \mathrm{X}_{01}{ }^{+\mathrm{h}}$. We note that the scheme here represents conventional, A-type blinking associated with Auger recombination, but that B-type PI can be introduced with the addition of two competing pathways for hot electrons produced by above band-edge excitation; the conventional internal conversion route to band-edge emission and a trapmediated, non-radiative transition to the ground-state.
The energetics of the CTST model is best rationalised by referencing the band structure of the QD and host medium (Fig. 2b). Here, we consider QD core, shell and matrix valence (VB) and conduction (CB) band-energies combine with interband trap-states to define reaction-coordinates for exciton-electron and hole-tunnelling, while the kinetic energies of the charge-carriers are determined by quantum confinement and the electron-hole Coulomb interaction. To first order, the tunnelling rate constants $k_{\mathrm{i}}^{+},{k_{\mathrm{i}}}^{-},{k_{\mathrm{h}}}^{+}$and ${k_{\mathrm{h}}}^{-}$can be defined by the general form ${ }^{49}$

$$
k=A \frac{\sigma}{4 \pi R_{0}^{2}} e^{(-l \sqrt{8 m(V-E)} / \hbar)}
$$

where, $A=2 E / h$, is the attempt-to-escape frequency of the electron or hole from the QD-core, surface-state or external trap, $l$ is the tunnelling length, $m$ the particle mass, $V$ the tunnelling 
(a)
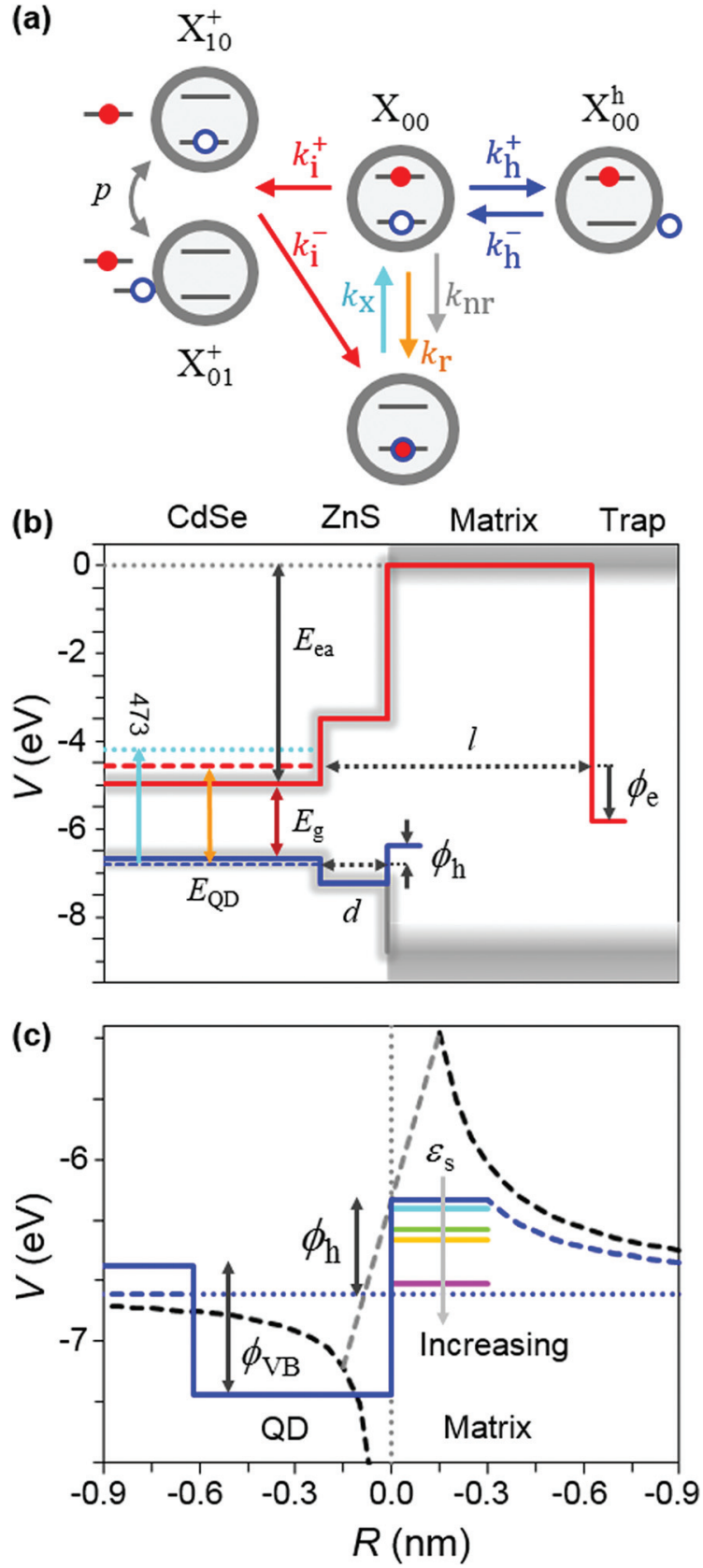

Fig. 2 Kinetics and energetics in CTST. (a) The five-state kinetics scheme, consisting of: a ground-state (bottom), exciton-core $\left(X_{00}\right)$, surface-hole, $\left(X_{00}{ }^{h}\right)$ and ionised charged-core $\left(X_{10}{ }^{+}\right)$and chargedsurface $\left(\mathrm{X}_{01}{ }^{+}\right)$exciton-states connected by excitation, emission and non-radiative rates constants, $k_{\mathrm{x}}, k_{\mathrm{r}}, k_{\mathrm{nn}}$, tunnelling rate constants $k_{\mathrm{i}}^{+}, k_{\mathrm{i}}^{-}$, $k_{\mathrm{h}}{ }^{+}, k_{\mathrm{h}}{ }^{-}$and probability $p$ that selects between ionised-states. (b) Bandenergy diagram. VB and CB energies of a CdSe QD-core, ZnS shell and vacuum and surface and host (pT) trap-states. Electron (red dash) and hole (blue dash) confinement energies are defined empirically (ESI eqn $(5) \dagger)$, while the QD-core, ZnS and vacuum band-offsets define electrontunnelling coordinate (solid red) over distance $l$ and hole tunnelling coordinate (solid blue) through shell thickness $d$. (c) Hole-trapping potentials for pT (blue), PS (cyan), $\mathrm{SiO}_{x}$ (green), PVP (orange) and PVA (magenta) due to electrostatic self-energies (black dash) at the dielectrically mismatched QD-host interface. Trap radius $r=0.3 \mathrm{~nm}$ (approximating the $\mathrm{ZnS}$ lattice spacing) is chosen to regularize the self-energy at the surface (grey dash). Hole (blue dash) confinement energy is also indicated for reference. potential and $E$ is the kinetic energy of the charge-carrier. The factor $\sigma / 4 \pi R_{0}^{2}$ represents a carrier-capture probability at a trap (or at the QD) with a cross-section $\sigma$ and QD-centre to trapcentre distance $R_{0}$ (note $\mathrm{S} 2$, eqn (S2) and (S3) and Fig. S2 in ESI†).

\section{Exciton and trap energetics}

In defining the trap-states that make up the charge-tunnelling reaction coordinates we assume the electron is stabilised by a potential, $\phi_{\mathrm{e}}$, below the QD-CB and the hole by a potential $\phi_{\mathrm{h}}$, above the QD-VB. In this case back-tunnelling is "uphill" in both cases, requiring thermal activation of the charge-carrier for which standard energies are generally insufficient $(k T=$ $0.026 \mathrm{eV}$ at room temperature). ${ }^{49}$ Instead, we assume the electron tunnels "downhill" to recombine with the excess-hole on the QD, either at the VB-edge in the core-state, $\mathrm{X}_{10}{ }^{+}$or at the surface-trap above the $\mathrm{VB}$ in $\mathrm{X}_{01}{ }^{+}$. In this case, mean barrier heights for tunnelling forward to $(+)$ and back from $(-)$ the host-trap are given by (note S3 in ESI $\dagger)^{50}$

$$
\begin{aligned}
& V_{\text {ion }}{ }^{+}=E_{\text {ea }}-\phi_{\mathrm{e}} / 2 \\
& V_{\text {off }^{-}}=E_{\text {ea }}+\phi_{\mathrm{e}}-\left(E_{\mathrm{QD}}-\phi_{\mathrm{e}}\right) / 2 \\
& V_{\text {on }^{-}}{ }^{-}=V_{\text {off }^{-}}+\phi_{\mathrm{h}} / 2
\end{aligned}
$$

where, $E_{\text {ea }}$ is the electron affinity of the semiconductor and $E_{\mathrm{QD}}$ is the QD band-gap (Fig. 2b). Based on experimental observations, we attribute the dielectric dependency of offtime blinking statistics to electron stabilisation in the host medium by a self-energy, $\phi_{\mathrm{e}}=\left(1-1 / \varepsilon_{\mathrm{m}}\right) e^{2} / 2 r_{\mathrm{e}}$ (Gaussian units) with electron return to the QD-core VB-edge. The slower decay of the on-time PDDs is associated with recombination of the electron with a hole stabilised at the QD surface by a selfenergy, $\phi_{\mathrm{h}}=\left(1 / \varepsilon_{\mathrm{s}}-1 / \varepsilon_{\mathrm{OD}}\right) K e^{2} / 2 r_{\mathrm{h}}$. Here $\varepsilon_{\mathrm{m}}, \varepsilon_{\mathrm{s}}$ and $\varepsilon_{\mathrm{QD}}$ define the dielectric constants of the host-matrix, the QD surface and the QD core respectively (eqn (S4) in ESI $\dagger$ ), ${ }^{51,52} K=\left(\varepsilon_{\mathrm{QD}}-\varepsilon_{\mathrm{S}}\right) /$ $\left(\varepsilon_{\mathrm{QD}}+\varepsilon_{\mathrm{s}}\right)$ is a screening factor due to the dielectric mismatch at the QD surface and $r_{\mathrm{e}}, r_{\mathrm{h}}$ are radii of the electron and hole trapping cavities. The self-energy at the QD-host interface is classically unbounded and $r_{\mathrm{h}}$ is a cut-off imposed to regularise the potential, such that the trap is represented by a linear extrapolation of the image-potential within a "lattice-spacing" either side of the interface (Fig. 2c). ${ }^{53}$ We found that a fixed radii, $r_{\mathrm{e}}=r_{\mathrm{h}}=r=0.3 \mathrm{~nm}$, reproduces QD-blinking statistics rather well, with good correlation between simulation and experiment. The trap dimension also compares well with the lattice spacing in the QD-capping material and typical void dimensions expected in the host matrices (note $\mathrm{S} 4$ in ESI $\dagger$ ). ${ }^{54-56}$ We note that, while the trap definitions given above are only zeroth order approximations based on simple Born solvation and the electrostatics of the QD-host interface, they serve to illustrate the dielectric dependencies of PI and allow the simple calculation of barrier heights along the tunnellingcoordinate.

For exciton-hole tunnelling between the QD-core and surface-states, we define a mean barrier, $V_{2 \mathrm{~h}}{ }^{ \pm}=\phi_{V \mathrm{~B}} \mp q \phi_{2 \mathrm{~h}}$, for forward $\left(V^{+}\right)$and back $\left(V^{-}\right)$processes, where, $\phi_{V \mathrm{~B}}$, is the core-to- 
shell valence band offset, $q$ is the excess charge on the QD and $\phi_{2 \mathrm{~h}}=e^{2} / \varepsilon_{\mathrm{S}} R_{\mathrm{c}}$ approximates the combination of Coulomb repulsive and charging potentials for two holes in the VB at a QDcore radius $R_{\mathrm{c}}{ }^{57,58}$ Importantly, in the ionised QD-state the barrier to forward-tunnelling of the exciton-hole to the QDsurface is lowered by $-\phi_{2 \mathrm{~h}}$, but raised by $+\phi_{2 \mathrm{~h}}$, to back-tunnelling to the QD-core. The effect is to shift the equilibrium, $\mathrm{X}_{01}{ }^{+}$ $\leftrightarrow \mathrm{X}_{01}{ }^{+\mathrm{h}}$, toward the non-radiative, surface-hole compared to the neutral-state equivalent process, $\mathrm{X}_{00} \leftrightarrow \mathrm{X}_{00} \mathrm{~h}$, with a subsequent reduction in PL yield. To summarise, the dielectric dependent tunnelling barriers within the CTST framework are determined by the QD band-energies and the electrostatics of solvation, dielectric interfaces and charged particulates (Table S2 in ESI $\dagger$ ). Other parameters of the CTST model are discussed below along with results from simulations.

\section{PL intensity trajectory simulations and PI statistics}

To complete the parameterisation of the electron-tunnelling rate constant, the electron affinity, $E_{\text {ea }}$, can generally be sourced from semiconductor literature $(\mathrm{CdSe}=4.95 \mathrm{eV}),^{59}$ while the charge-carrier kinetic energy, $E$, is determined from $E_{\mathrm{QD}}$, the bulk CdSe band-gap, $E_{\mathrm{g}}$, and the exciton-pair Coulomb energy (eqn (S5) and Fig. S3 in ESI†). For the CdSeZnS QDs (Lumidot 590, Sigma-Aldrich, UK) used in our experi- ments, $R_{\mathrm{c}}=2 \mathrm{~nm}$ and the electron and hole confinement energies are $0.39 \mathrm{eV}$ and $0.11 \mathrm{eV}$ respectively. The excitation rate constant, $k_{\mathrm{x}}=\sigma_{\mathrm{x}} \lambda I / h c$, for a wavelength, $\lambda$ and intensity, $I$ is parameterised through a size-dependent absorption crosssection, $\sigma_{\mathrm{x}}$, along with relaxation constants $k_{\mathrm{r}}$ and $k_{\mathrm{nr}}$ (note $\mathrm{S} 6$ and eqn (S6), (S7) and (S8) in ESI $\dagger$ ). ${ }^{60-62}$ Finally, motivated by the sensitivity of blinking to passivation of the QD surface, we make the probability of finding the QD in the surface charged state, $\mathrm{X}_{01}{ }^{+}$, following ionization equal to the surface fraction of atoms within the spherical cluster approximation, i.e., $p=4 N^{-1 / 3}$ for an $N$ atom QD and $N^{-1 / 3}=a / R_{\mathrm{s}}$, where $R_{\mathrm{S}}$ is the $\mathrm{QD}$ core + shell radius and $a$ is the radius of the atoms making up the cluster. For simplicity we approximated $a$ to half the CdSe bond-length $(0.5 \times 0.26 \mathrm{~nm}) .{ }^{63}$ In this model $p$ represents a ratio of surface-to-core hole-traps on a per atom basis, but other models of $p$ can be envisaged, where surface modification or core-dopants may bias the fraction of surface traps. For a typical $\mathrm{ZnS}$ shell of 2 monolayers (ML) and lattice spacing $0.31 \mathrm{~nm}, R_{\mathrm{S}}=2.62 \mathrm{~nm}$ and the hole trapping probability, $p=0.2$, compares favorably with models elsewhere. ${ }^{20}$ Blinking statistics generated from simulated single QD PL intensity trajectories shows TPL behaviour in the log-log PDDs of off- and on-times is reproduced strikingly well by the CTST model (Fig. 3a and 3b), along with the same trend in intensi-

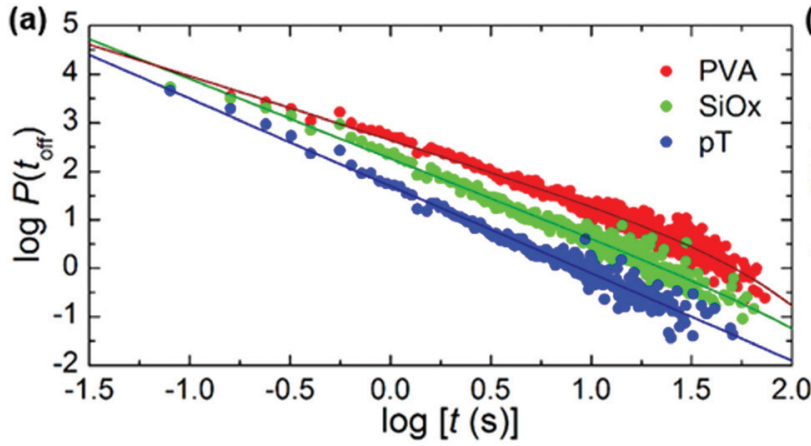

(c)
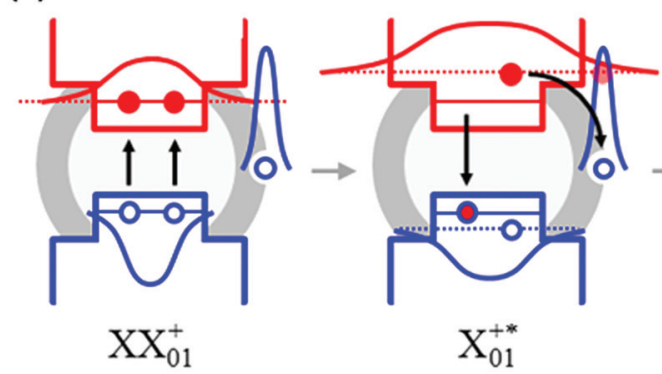

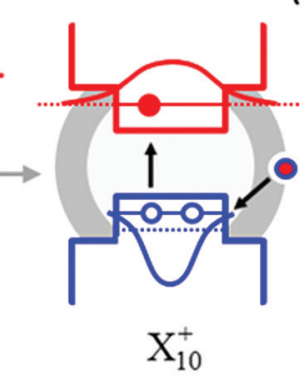

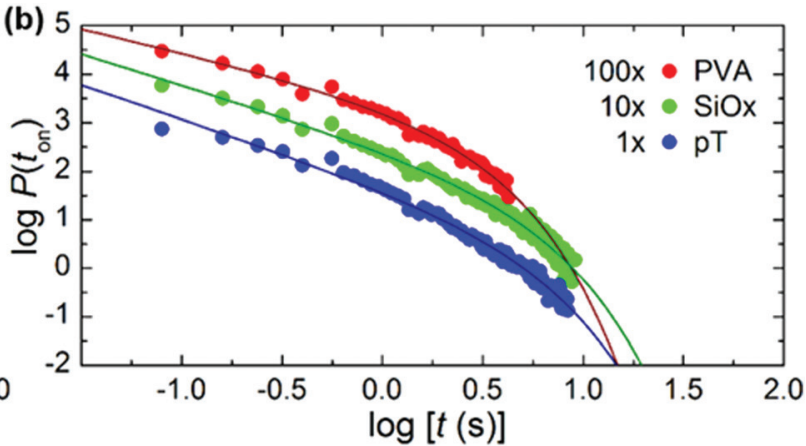

(d)

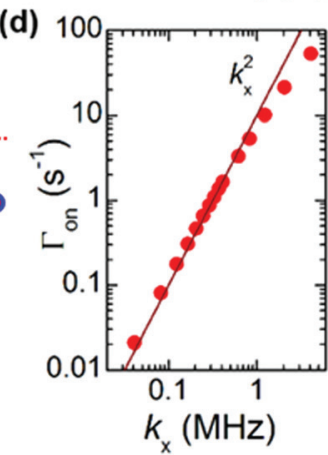

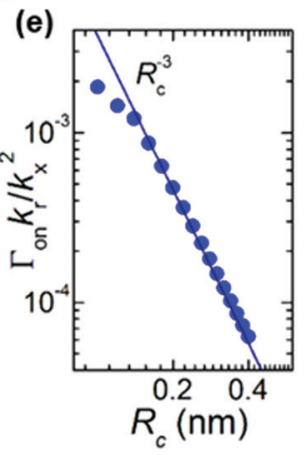

Fig. 3 Simulated QD blinking statistics and surface-to-core charge switching mechanism. (a) Log-log PDDs of off-time and (b) on-times from simulated PL trajectories in different dielectric media along with corresponding TPL fits (lines) with decay, $\alpha$ (on/off) and cut-off, $\tau_{\mathrm{c}}$ (on/off) parameters defined in ESI Table S3. $\uparrow$ On-time PDDs are scaled for clarity. CTST input parameters are: $R_{\mathrm{c}}=2.0 \mathrm{~nm} ; d=0.62 \mathrm{~nm} ; \sigma_{\mathrm{x}}=1.72 \times 10^{-15} \mathrm{~cm}^{2}, I=$ $45 \mathrm{~W} \mathrm{~cm}^{-2}$ and $\lambda=473 \mathrm{~nm}$ give $k_{\mathrm{r}}^{-1}=23 \mathrm{~ns} ; k_{\mathrm{nr}}=2.85 \times 10^{10} \mathrm{~s}^{-1}$. (c) Mechanism for the origin of saturation in the on-time PDD. A surface-charged biexciton $\mathrm{XX}_{01}{ }^{+}$undergoes Auger relaxation to excited the $\mathrm{X}_{01}{ }^{+*}$-state with a hot-electron that recombines at the surface-localised excess-hole to leave charge on the QD-core in the $\mathrm{X}_{10}{ }^{+}$-state. (d) Excitation rate dependence of the cut-off rate, $\Gamma$, from simulation (points), along with the $k_{\mathrm{x}}{ }^{2}$ (line) dependency of the biexciton formation rate. (e) QD-core size dependence of the electron-hole recombination probability from simulation (points) and $R_{\mathrm{c}}{ }^{-3}$ (line) dependency of the localised surface-hole to core-electron volume ratio. 
ties, from PL-on to PL-off with increasing $\varepsilon_{\mathrm{m}}$ (Fig. S4 in ESI $\dagger$ ), as that observed experimentally (Fig. 1a). Simulation and experimental parameters were matched as closely as possible (note $\mathrm{S} 7$ in ESI $\dagger$ ), in particular the CdSe core-size $\left(R_{\mathrm{c}}=2 \mathrm{~nm}\right.$ ), shell-thickness (2 ML) and surface-ligand (hexadecylamine, HDA, $\varepsilon_{\text {lig }}=2.7$ ) were defined by the CdSe-ZnS QD source (Lumidot 590, Sigma-Aldrich, UK). ${ }^{64}$

To model saturation in the TPL at long on-times, we introduce the rate constant, $\Gamma=\tau_{\mathrm{c}}{ }^{-1}$, for quenching of the radiative $\mathrm{X}_{01}{ }^{+}$-state. Despite its ubiquity in QDs, nanorods and other PL materials, the mechanism for truncation in the power-law is not wholly understood. Indeed, truncation can be an artefact of the data analysis, where on-off events are under-sampled. ${ }^{65}$ Motivated by previously observed dependencies of $\tau_{\mathrm{c}}$ on excitation-rate, QD size, ${ }^{28}$ the dielectric properties of the nanoenvironment ${ }^{31}$ and temperature, ${ }^{5}$ we propose the following simple mechanism for quenching of $\mathrm{X}_{01}{ }^{+}$(Fig. 3c). Given the QD-core is neutral in the surface-charged state, the biexciton $\mathrm{XX}_{01}{ }^{+}$will be formed at a rate approximating to $k_{\mathrm{xx}}=k_{\mathrm{x}} f_{\mathrm{c}} / 2$, where $f_{\mathrm{c}} \sim k_{\mathrm{x}} /\left(k_{\mathrm{x}}+k_{\mathrm{r}}\right)$ is the core-exciton fraction (eqn (S1) in ESI $\dagger)$. The biexciton will decay rapidly by Auger recombination with near-unit probability, $k_{\mathrm{nr}} /\left(k_{\mathrm{nr}}+k_{\mathrm{r}}\right) \approx 1$, with the excess band-gap energy creating a hot-electron state, $\mathrm{X}_{01}{ }^{+}$, that is highly delocalised, enveloping the QD-surface and the deeptrapped excess-hole. Assuming electron-hole recombination is strongly modulated by the electron density at the localised surface-hole or core exciton-hole, then the probability of recombination at the surface will scale with the core-to-trap volume ratio as $\left[1+\left(R_{\mathrm{c}} / r\right)^{3}\right]^{-1}$. Thus, for hole quenching that is rate-limited by biexciton formation, the cut-off rate is simply approximated by $\Gamma=\left(k_{\mathrm{x}}^{2} / 2 k_{\mathrm{r}}\right) \cdot\left(r / R_{\mathrm{c}}\right)^{3}$, for low excitation intensities $\left(k_{\mathrm{x}} \ll k_{\mathrm{r}}\right)$ and a small hole-trap $\left(r \ll R_{\mathrm{c}}\right)$. The cut-off rate, in this case, has the correct squared dependency on the excitation rate and the inverse-cubic dependency on QD size (Fig. 3d and $3 e$ ) reported elsewhere (within error). ${ }^{28}$ Less clear, is how the observed sensitivity of $\tau_{\mathrm{c}}$ to $\varepsilon_{\mathrm{m}}$ can be accounted for. Here, we recognize that since the rate constants, $k_{\mathrm{x}}$ and $k_{\mathrm{r}}$ are dependent on the square of the local field factor, $F=3 \varepsilon_{\mathrm{s}} /\left(2 \varepsilon_{\mathrm{s}}+\varepsilon_{\mathrm{QD}}\right)$, so too will $\Gamma$, since $\varepsilon_{\mathrm{s}}$ is a composite of host and ligand dielectric constants (eqn (S4) in ESI $\dagger$ ). The complete expression describing the full set of dependencies of the on-time cut-off rate in our model is given by

$$
\Gamma_{\text {on }}=\frac{k_{x}}{2 k_{r}}|F|^{2}\left(\frac{r}{R_{c}}\right)^{3} e^{-E_{a} / k T}
$$

where the Arrhenius term describes the temperature dependence of the on-time saturation with activation energy $E_{\mathrm{a}} \sim$ $0.003 \mathrm{eV}$, derived from experimental data in the literature $\left(\tau_{\mathrm{c}} \sim\right.$ $164 \mathrm{~s} @ T=10 \mathrm{~K}$ and $\left.\tau_{\mathrm{c}} \sim 7 \mathrm{~s} @ T=300 \mathrm{~K}\right) .{ }^{5,21}$ For completeness, a rate constant for the slow quenching of $\mathrm{X}_{10}{ }^{+}$is introduced to account for saturation in the off-time PDD. Typically this occurs an order of magnitude or so beyond the on-time cut-off and we simply attribute the lower saturation rate, $\Gamma_{\text {off }}=\left(k_{\mathrm{x}}^{2}\right)$ $\left.2 k_{\mathrm{r}}\right) \cdot|F|^{2}$ to a reduced rate of biexciton formation in the presence of QD-core charge and Auger quenching. The exact form of the expression does not appear critical to modelling the overall blinking statistics in our experiments, possibly due to the extended saturation in off-times (up to $50 \times$ longer than ontimes) reducing the interdependency between on- and off-time statistics. We note that cut-off times may also be limited by a finite charge-tunnelling length and that the subtle interplay between decay and truncation in the on/off PDDs remains the subject of ongoing investigation. Given the large uncertainties in both experimental and simulated values of cut-off times, no further justification is provided here for the form of $\Gamma_{\text {off }}$ other than providing the correct "order-of-magnitude" value observed in our experiments and elsewhere. ${ }^{31}$

We tested the CTST model against our observed dependencies of $\alpha_{\text {on }}, \alpha_{\text {off }}$ and $\tau_{\mathrm{c}}$ (on) on the host dielectric constant, $\varepsilon_{\mathrm{m}}$, by fitting the TPL function to on- and off-time PDDs derived from simulated PL trajectories (Table S3 in ESI $\dagger$ ). Correlations of both $\alpha_{\text {on }}$ and $\alpha_{\text {off }}$ with the reaction field $\left(1-1 / \varepsilon_{\mathrm{m}}\right)$ were found comparable to those observed experimentally (Fig. 4a and $4 \mathrm{~b}$ ) and consistent with the general conclusion that deeper charge-trapping in more polarizable and dielectrically mismatched media leads to longer sojourn-times in bright and dark-states. Furthermore, the strong linear correlation of the experimental on-time cut-off, $\tau_{\mathrm{c}}=\Gamma_{\mathrm{on}}{ }^{-1}$, with $\varepsilon_{\mathrm{m}}{ }^{-1}$ is closely-reproduced by the CTST model (Fig. 4c). Given the simplicity of the model the comparisons are striking. In particular, the same weak trend in $\alpha_{\text {on }}$ with the reaction field is observed in both our experimental data and simulations. This can be rationalised in CTST by a surface-hole trapping-potential $\left(K / \varepsilon_{\mathrm{m}}\right)$ that opposes the reaction field $\left(1-1 / \varepsilon_{\mathrm{m}}\right)$ trapping the electron in the host. This produces a tunnelling-barrier, $\mathrm{V}_{\mathrm{on}}{ }^{-}$, for electron-recombination in $\mathrm{X}_{01}{ }^{+}$that is only weakly dependent on $\varepsilon_{\mathrm{m}}$. Conversely recombination in $\mathrm{X}_{10}{ }^{+}$occurs at the VB-edge of the $\mathrm{QD}$, where the barrier $\mathrm{V}_{\text {off }}{ }^{-}$remains strongly dependent on $\varepsilon_{\mathrm{m}}$, through the reaction field of the host.

\section{Intensity-spikes and charge dependent grey-state emission}

To understand the influence of exciton charge-carrier dynamics on the modulation of PL in QD blinking, we examined the intensity histogram for the appearance of intermediate emission levels or so-called "grey"-states. ${ }^{6,18}$ Histograms for the range of dielectric support media we examined are characterised by a bimodal distribution of PL intensities and typified by CdSe-ZnS QDs in pT (Fig. 5a). Dark and brightstates approximate to two, normal-like distributions with standard deviations characteristic of the detection-noise, as well as a variance due to on/off blinking within the photon integration time. Resolving intermediate states by fitting more than two normal distributions cannot be easily justified from the sum histogram in this case. However, simulation of the QD PL trajectory using CTST allows resolution of the intensity histogram into dark, bright and intermediate intensity distributions (Fig. 5b). By tracking charge on the QD-core $\left(q_{\mathrm{c}}\right)$ and surface $\left(q_{\mathrm{s}}\right)$ and the net charge $\left(q=q_{\mathrm{c}}+q_{\mathrm{s}}\right)$ on the QD, intensities corresponding to the dark, $\mathrm{X}_{10}{ }^{+}$-state $\left(q_{\mathrm{c}}=1\right)$ and radiative, $\mathrm{X}_{01}{ }^{+}$-state $\left(q_{\mathrm{s}}=1\right)$ can be separated from intermediate intensities arising from mixed contributions from the ionised states 
(a)

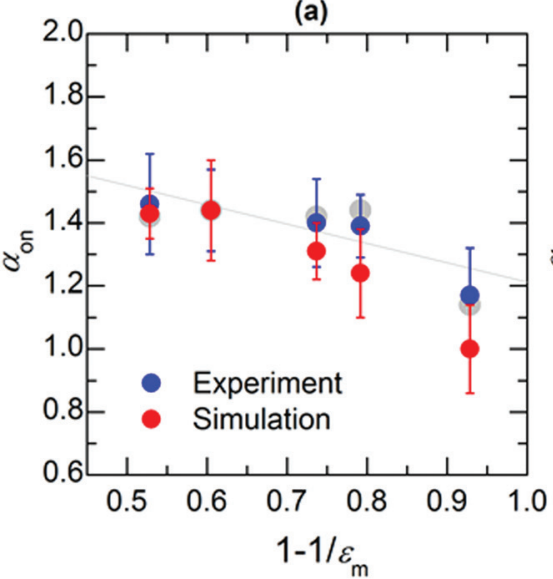

(b)

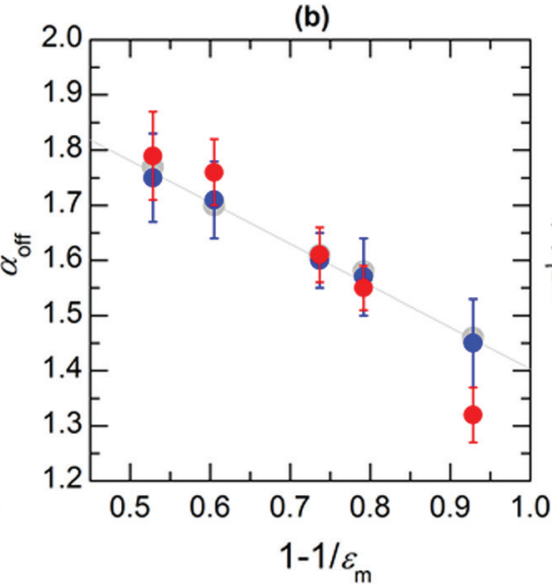

(c)

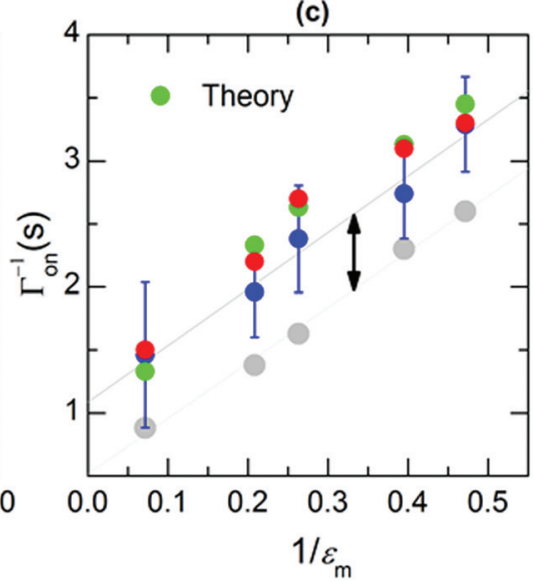

Fig. 4 CTST simulation vs. experimental and TPL cut-off time size dependency. Dependence of (a) $\alpha_{\mathrm{on}}$ and (b) $\alpha_{\mathrm{off}}$ and (c) the cut-off time, $\tau_{\mathrm{c}}$ (on) $=$ $\Gamma^{-1}$, on the dielectric constant $\varepsilon_{\mathrm{m}}$ of different host-media. Plotted are mean values from fitting the TPL to simulated PL trajectories along with experimental values. Error bars correspond to standard deviations in data sets from over 25 simulations per host. Values from ref. 31 (grey) and linear fits to experimental points are plotted for comparison. Theoretical values in (c) for $\tau_{\mathrm{c}}$ (on) are from eqn (3) and the separation of linear fits (arrow) correlates with the QD core-size difference between our experiments and that of ref. $31\left(R_{\mathrm{c}} \sim 1.7 \mathrm{~nm}\right)$.

and the neutral, $\mathrm{X}_{00}$-state, with a net QD charge less than unity $(q<1)$. For the QD-type (Lumidot 590, Sigma-Aldrich, UK) studied here, $\mathrm{X}_{01}{ }^{+}$dominates $(70 \%)$ the bright state population with a smaller contribution from mixed states (30\%). In this instance, emission from $\mathrm{X}_{01}{ }^{+}$is lowered from the native, $\mathrm{X}_{00}$-state by only a small (15\%) shift in the equilibrium, $\mathrm{X}_{01}{ }^{+}$ $\leftrightarrow \mathrm{X}_{01}{ }^{\text {th }}$ towards the non-radiative, surface-hole population compared to the native-state equivalent, $\mathrm{X}_{00} \leftrightarrow \mathrm{X}_{00}{ }^{\mathrm{h}}$. In addition, correlation between the net-charge and intermediate intensities shows the QD spends, at most, only half the photon integration time in $\mathrm{X}_{00}$ and half in $\mathrm{X}_{10}{ }^{+}$or $\mathrm{X}_{01}{ }^{+}$(Fig. $5 \mathrm{c}$ and $5 d)$. It thus appears the QD is rarely in its neutral, radiativestate for long. Indeed, by tracking the accumulated time spent in $\mathrm{X}_{00}(q=0)$, the QD is observed to settle rapidly into spending only $15 \%$ of its time undergoing PL from the native, emissive state (Fig. 5e).

The CTST model predicts that shorter photon integration times, should capture a greater contribution of $\mathrm{X}_{00}$ in intermediate emission levels, while QDs with thicker shells will increase the modulation depth between $\mathrm{X}_{00}$ and $\mathrm{X}_{01}{ }^{+}$in the PL intensity trajectory. To test this prediction, we performed experiments on CdSe-ZnS QDs (EviDot, birch yellow, em: $580 \mathrm{~nm}$, core size $3.2 \mathrm{~nm}$, Evident Tech. Inc., USA) in PVA at fast image acquisition rates ( $40 \mathrm{fps}$ ). Observation of transient "spikes" in the PL intensity trajectory of this QD-host system have been reported elsewhere, but with only qualitative explanation. ${ }^{6}$ We found QD trajectories that displayed three distinct levels of emission: high intensity "spikes"; a steady-state "grey"-level; and the common dark-state (Fig. 5f). Although, these states are not fully resolved in the intensity histogram, the bright-state population does exhibit a broad-tail that extends beyond the normal distribution-width expected for detection-noise (Fig. 5g). Again, simulation of the PL trajectory with a correct CdSe-core size $\left(R_{\mathrm{c}}=1.6 \mathrm{~nm}\right)$ and thick ZnS-shell $(d=1.8 \mathrm{~nm})$ shows qualitative agreement with experiment
(Fig. $5 \mathrm{~h})$. We note the cap size (6 ML) exceeds the average thickness (3-4 ML) from our TEM measurements (Fig. 5g inset) and those reported elsewhere ${ }^{66}$ but is consistent with the relatively low number of QDs found to exhibit "spikes" and "greys" in their PL trajectory traces. Importantly, resolution of the intensity histogram into core-charged $\left(q_{\mathrm{c}}=1\right)$, surfacecharged $\left(q_{\mathrm{s}}=1\right)$ and non-integer net charge $(q<1)$ populations, as well as the correlation between the net-charge and intensity, shows the highest PL intensities correspond, in this case, to emission from the native, neutral state of the QD (Fig. 5i). The results indicate that $\mathrm{X}_{01}{ }^{+}$is the "grey"-state that appears as the "bright"-state for thin-shelled QDs, with the neutral $\mathrm{X}_{00}$-state being sampled only transiently (Fig. 5j). With increasing shell thickness, the "grey"-state is resolved at decreasing levels of emission compared to the neutral-state "spikes". The CTST model thus provides a compelling origin for the "grey"-state. Given the equilibrium constant, $k_{\mathrm{h}}{ }^{+} / k_{\mathrm{h}}{ }^{-}$is governed by tunnelling of the exciton-hole through the QDshell, it will be modulated by the thickness of the capping layer. However, the tunnelling barriers, $\mathrm{V}_{2 \mathrm{~h}}{ }^{ \pm}=\phi_{\mathrm{VB}} \mp q \phi_{2 \mathrm{~h}}$ and consequently the equilibrium constants are markedly different for $\mathrm{X}_{00}$ $(q=0)$ and $\mathrm{X}_{01}{ }^{+}(q=1)$; the latter having a stronger dependency on shell thickness $d$, which pushes the equilibrium, $\mathrm{x}_{01}{ }^{+} \leftrightarrow$ $\mathrm{X}_{01}{ }^{\text {th }}$, towards the non-radiative exciton-hole surface-state with increasing cap-depth. As $d$ increases, PL intensities from $\mathrm{X}_{00}$ and $\mathrm{X}_{01}{ }^{+}$diverge with the latter quenching rapidly with the cap depth and ultimately resolving as a distinct "grey"-state in the PL trajectory of the QD (Fig. 5k and Fig. S5 in ESI†).

\section{Grey-state dependent PL enhancement and decay}

To understand the role of exciton charge-carrier dynamics in QD brightening and bleaching we examined single QDs under oxidative conditions. For CdSe-ZnS core-shell QDs (EviDot, fort orange, em: $600 \mathrm{~nm}$, size $4.0 \mathrm{~nm}$, Evident Tech. Inc, USA) on $\mathrm{SiO}_{x}$, under moderate-to-high excitation intensities $\left(>500 \mathrm{~W} \mathrm{~cm}^{-2}\right)$ 

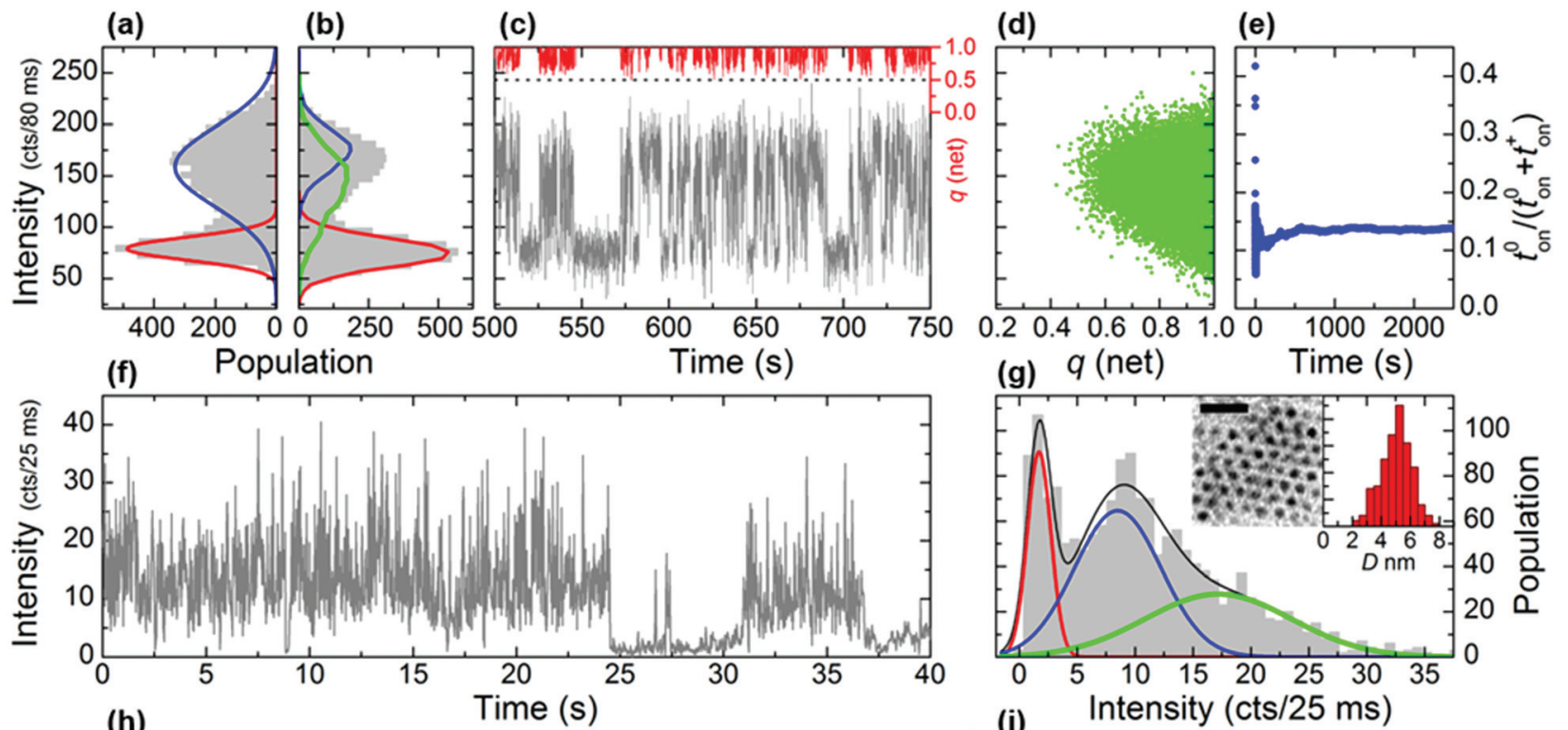

(h)

Time (s)

(i)
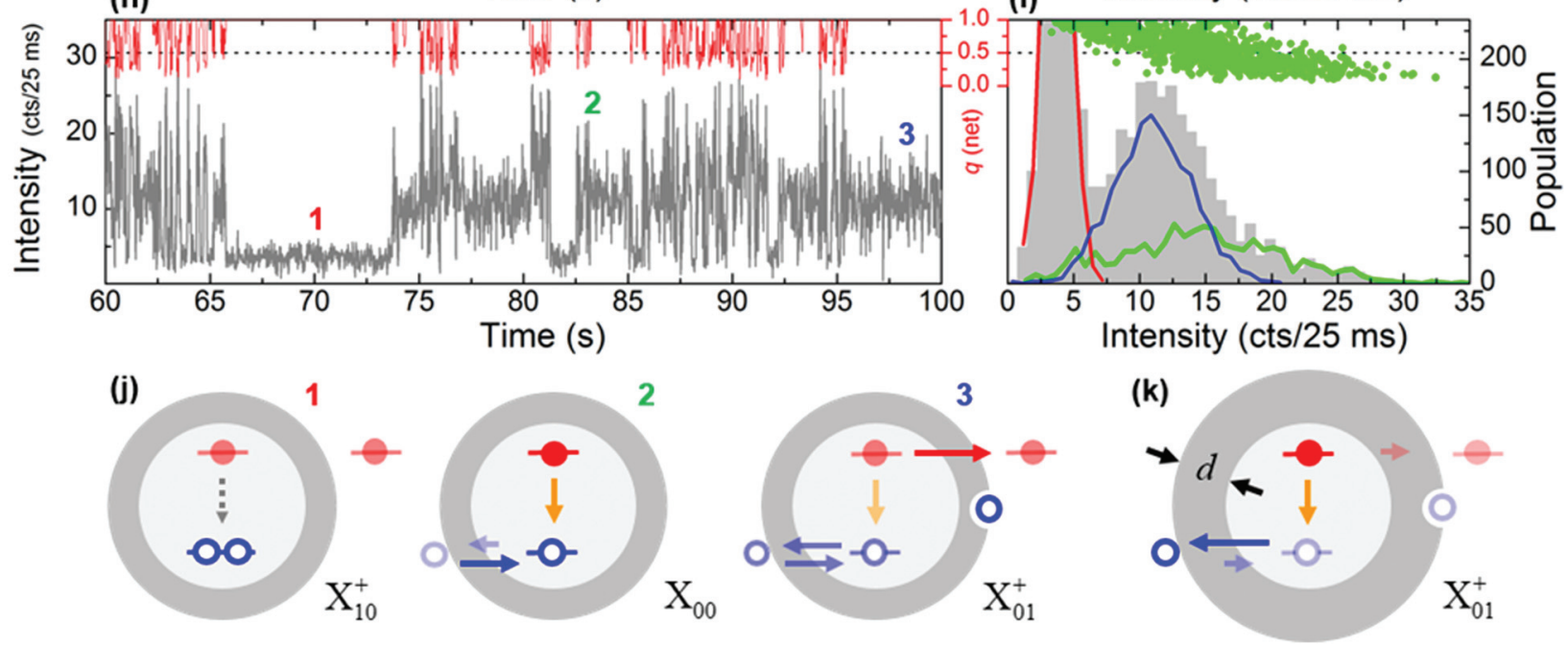

Fig. 5 PL intensity analysis and grey-state resolution in the CTST simulation. (a) Intensity histogram from a typical PL intensity trajectory of a CdSeZnS QD in pT (Fig. 1a) along with a fit of two normal distributions to dark (red) and bright (blue) populations. (b) as (a) but from a simulated QD trajectory (c), showing resolution of dark (red, $q_{c}=1$ ), intermediate (green, $q_{c}+q_{s}<1$ ) and bright-state (blue, $q_{s}=1$ ) intensity populations. (c) Extract from a simulated PL trajectory (grey) for CdSe-ZnS QD in pT $\left(\varepsilon_{\mathrm{m}}=2.12\right)$ with CTST parameters as for Fig. $3 a$. Inset is the net charge, $q$ (red) on the QD (per time bin). (d) Correlation between net QD charge $(q<1)$ and intermediate PL intensities for a full trajectory of 2500 on/off events $(2675 \mathrm{~s})$ showing the QD-charge rarely drops below 0.5. (e) Plot of the time spent in the neutral, $X_{00}$-state, as a fraction of the total time in emissive states, $\mathrm{X}_{00}+\mathrm{X}_{01}{ }^{+}$showing the QD spends only $15 \%$ of its time in the native state. (f) Experimental PL intensity trajectory of a CdSe-ZnS QD (EviDot 580) in PVA showing steady-state, dark and intermediate "grey"-states and transient intensity-"spikes". (g) Intensity histogram showing resolution of the dark (red), grey (blue) and bright (green) states from fitting three normal distributions. Inset: TEM of CdSe-ZnS QDs (20 nm scale bar) and corresponding size distribution from variation in cap and core growth. (h) Simulated PL intensity (grey) and net QD charge (red) for a CdSe-ZnS QD with a $1.6 \mathrm{~nm}$ core and $2 \mathrm{~nm}$ shell. (i) Intensity histogram showing resolution of the dark (red, $q_{\mathrm{c}}=1$ ), intermediate-"grey" (blue, $q_{\mathrm{s}}=1$ ) and bright (green, $q<1$ ) state populations. (j) Schematic showing exciton processes in the QD, for (1) Auger quenching of the core-trion, $\mathrm{X}_{10}{ }^{+}$in the dark-state, (2) weak modulation of the core exciton-hole population in the bright, native $X_{00}$-state and (3) suppression of core exciton-hole population in the "grey"$\mathrm{X}_{01}{ }^{+}$state. (k) For QDs with thicker-shells, electron-tunnelling from $\mathrm{X}_{00}$ is suppressed, but the exciton-hole tunnelling equilibrium, $\mathrm{X}_{01}{ }^{+} \leftrightarrow \mathrm{X}_{01}{ }^{+h}$, tends towards the surface hole-state with lower "grey"-level emission.

many PL intensity trajectories displayed an envelope of QY enhancement and decay over time (Fig. 6a). By way of control, interrogation of bare CdSe QDs showed only PL decay in the intensity trajectory, with no evidence of PE in the core-QDS sampled (Fig. 6a inset). Furthermore, the PE observed in our experiments appears predominantly irreversible in nature, as evidenced by a lack of dark-state recovery during periods of paused illumination (Fig. S6 in ESI†). To gain insight into the origin of PE we introduced a "chemically" modified QD state into the CTST kinetic scheme. Based on previous observations of photobleaching and bluing in QDs, ${ }^{11,42}$ as well as evidence of Se loss from CdSe QDs through $\mathrm{SeO}_{2}$ formation at the QD 

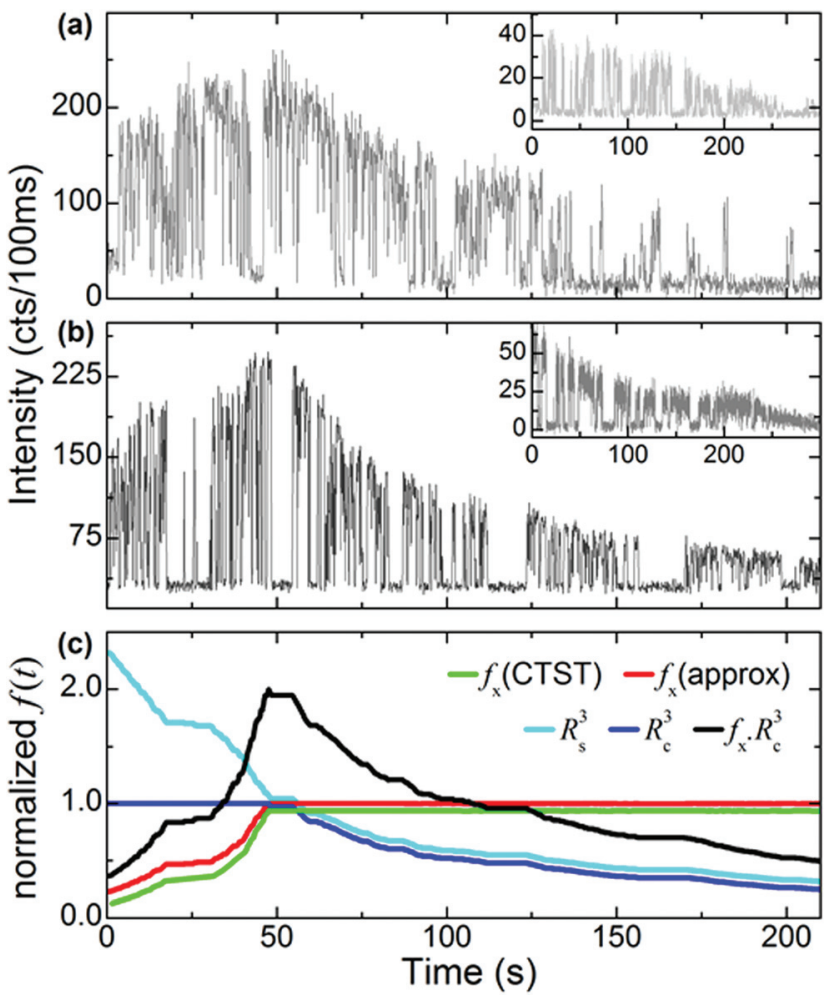

Fig. 6 Irreversible PL enhancement (PE) and decay. (a) Experimental PL intensity envelope of PE and photobleaching of a ZnS capped CdSe (EviDot 600) QD on glass $\left(\mathrm{SiO}_{\mathrm{x}}\right)$ under continuous illumination with $0.65 \mathrm{~kW} \mathrm{~cm}^{-2}$ at $473 \mathrm{~nm}$ and (inset) as synthesized uncapped CdSe $\left(R_{\mathrm{c}}=\right.$ $1.6 \mathrm{~nm})$ QDs. (b) Simulated PE and decay of the capped and (inset) uncapped QDs within the CTST framework and an atomistic model of $Q D$ degradation (see text) with a quantum yield $\Phi=5 \times 10^{-5}$. Parameters were closely matched to experiment as per Fig. 3 for the CdSe-ZnS $\left(R_{\mathrm{c}}=\right.$ $2 \mathrm{~nm}$ core and $2 \mathrm{ML}$ shell) with excitation intensity at $0.6 \mathrm{~kW} \mathrm{~cm}$. Uncapped CdSe (inset) was simulated for as synthesized $560 \mathrm{~nm}$ emitting QDs $\left(R_{\mathrm{C}}=1.6 \mathrm{~nm}\right.$ core and $0 \mathrm{ML}$ shell) on glass with other parameters as per Fig. 3. (c) Separation of the components contributing to $\mathrm{PE}$ and PL decay including; (green) the fractional population available for emission, $f_{\mathrm{x}}=f_{\mathrm{c}} /\left(f_{\mathrm{c}}+f_{\mathrm{s}}\right)$, from both simulation; (red) a simple approximation using the core-surface equilibrium constant, $f_{\mathrm{x}} \propto(1+$ $\left.k_{\mathrm{h}}{ }^{+} / k_{\mathrm{h}}{ }^{-}\right)^{-1}$; (blue) the degradation of the core and (cyan) core + shell volumes; (black) the overall effect of the changing emissive fraction and core volume reduction on the PL yield of a capped QD.

surface ${ }^{67}$ we associate the modified QD-state with degradation of the QD volume. To estimate the degradation rate, we refer to previous thin film and single molecule studies of QDs exposed to oxygen, ${ }^{7,37,68}$ which place the QY of PL activation in the region of $10^{-6}$ to $10^{-8}$ (QDs per excitation). In the simplest model we assume that degradation of the QD, regardless of mechanism, corresponds to the loss of "ion-pair" units (ZnS from the shell and CdSe from the core). In this case, given approximately, $N=\left(R_{\mathrm{s}} / a\right)^{3} \sim 8000$ atoms in a $2 \mathrm{~nm}$ CdSe core + $2 \mathrm{ML} \mathrm{ZnS}$ shell of sum radius, $R_{\mathrm{S}}=2.62 \mathrm{~nm}$ and a mean ion radius of $a=0.13 \mathrm{~nm}\left(\mathrm{Zn}^{2+}+\mathrm{S}^{2-}+\mathrm{Cd}^{2+}+\mathrm{Se}^{2-}\right)$, the low-end PE yield equates to an ion-pair loss of $\Phi=4 \times 10^{-5}$ per excitation (per QD). We simulate PL intensity trajectories with a rate constant, $k_{\mathrm{d}}=k_{\mathrm{x}} \Phi$, for transition from the exciton-state to the "chemically" degraded state and a modified QD radius, $R=$ $\left(R_{\mathrm{s}}{ }^{3}-2 a^{3}\right)^{1 / 3}$, following transition. Here, $R$ changes during degradation from the core + shell radius for $R_{\mathrm{s}}>R_{\mathrm{c}}$, to the core radius for $R_{\mathrm{S}}=R_{\mathrm{c}}$, following complete removal of the cappinglayer. While the model is crude, simulations of the PL enhancement and decay envelope compare well with typical experimental profiles under comparable conditions and within stochastic limits (Fig. 6b). Significantly, the QY of PE derived from simulation in this case $\left(\Phi=5 \times 10^{-5}\right)$, is closely matched to our low-end estimate, although we note that degradation yields can vary between individual QDs, as much as PL activation yields appear to vary in the literature. Such variation will reflect homogeneity in the QD nanoenvironment, as well as differences in the integrity of QD cap and core structures. Indeed, the model of PE presented here, inherently assumes that lattice reorganisation and relaxation is rapid following each degradation step, such that changes in PL intensity depend solely on the changes in the exciton-dynamics associated with a continuous QD core- and shell-size reduction, rather than the formation of vacancy trap-states. On the other hand, where structural rearrangement is slow, the latter will likely affect blinking and grey-state intensity modulation in a more discrete, quantized manner through multiple charging effects, for example. ${ }^{13}$ For core-only CdSe, experimentally observed PL decay appears marginally slower than predicted from simulation (Fig. 6b inset), which may result from the presence of an effective shell from residual oxide formation at the QD surface or merely from an overly simplified model of the degradation volume and/or estimate of the QY of the process. Nevertheless, the gross features of PE (and decay) that we attribute below to changes in the exciton dynamics, greystate emission levels and size dependent absorption, will be qualitatively similar irrespective of the model.

Interestingly, while PL decay is an obvious consequence of QD-core volume degradation from the approximate $R_{\mathrm{c}}{ }^{3}$ dependence of the absorption cross-section, the connection to PL enhancement is not so transparent. However, since exciton charge-carrier tunneling-rates are dependent on both size of the QD-core (via confinement energies) and shell thickness (via tunnelling distance), the kinetics of electron ionization and exciton-hole sampling of the QD-core and surface will be strongly modulated by changes in shell thickness and core radius. Specifically, since the fractional populations of the emitting states, $\mathrm{X}_{00}$ and $\mathrm{X}_{01}{ }^{+}$, are dependent on the exciton-hole equilibrium constant, $k_{\mathrm{h}}{ }^{+} / k_{\mathrm{h}}{ }^{-}$, between surface and core-states, the emission intensity will be strongly modulated by thinning of the capping layer. Given the barrier to forward-tunnelling in the equilibrium, $\mathrm{X}_{01}{ }^{+} \leftrightarrow \mathrm{X}_{01}{ }^{+\mathrm{h}}$, is lower than for back-tunnelling (due to hole-hole repulsion), $k_{\mathrm{h}}{ }^{+}$will rise more slowly than $k_{\mathrm{h}}{ }^{-}$ with decreasing shell-depth $d$, shifting the equilibrium toward the radiative core exciton-state, $\mathrm{X}_{01}{ }^{+}$. Quantitatively, it can be shown that the equilibrium constant approximates to $k_{\mathrm{h}}{ }^{+} / k_{\mathrm{h}}{ }^{-} \propto \exp \left(\phi_{2 \mathrm{~h}} \sqrt{\phi_{\mathrm{VB}}} d\right)$ for $\phi_{2 \mathrm{~h}}$ and $\phi_{\mathrm{VB}}$ in $\mathrm{eV}$ and $d$ in $\AA$ (note S8 and eqn (S9) in ESI $\dagger$ ), such that the core-emissive fraction, $f_{\mathrm{x}} \propto\left[1+k_{\mathrm{h}}{ }^{+} / k_{\mathrm{h}}{ }^{-}\right]^{-1}$ increases exponentially with decreasing cap thickness $d$ (Fig. 6c). The envelope of PL 
enhancement and decay can then be understood in the simplest sense, as a combination of an increasing core-exciton fraction $f_{\mathrm{x}}$ as $R_{\mathrm{S}}$ degrades towards $R_{\mathrm{c}}$ and a decreasing absorption "volume", $R_{\mathrm{c}}{ }^{3}$, with degradation of the QD-core.

\section{Conclusions}

In sum, the results support a broad description of QD photodynamics including blinking, grey-state emission and PL enhancement through CTST controlled exciton dynamics. The agreement between experimental and simulated data across a spectrum of key features in the PL intensity trajectory of single QDs is compelling, given the simple, rational assumptions applied in the model. In particular, experimental measurements of the QD blinking statistics, grey-state emission levels, intensity histograms and the envelope of irreversible PL enhancement and decay have been reproduced with good quantitative accuracy, within the limits of stochastic simulation. Most significantly is that these properties, which have previously been treated in isolation, have a clear and common origin within the CTST framework through the interplay between QD-core, surface and external host-states of the exciton charge-carriers. Evidently, the CTST basis describes exciton dynamics in an "average" QD, in a "uniform" dielectric medium and any "variance" in behaviour, due to heterogeneities in QD synthesis, the host, or engineered-states in the QDcore, surface or host, will require extension of the model with new or modified definitions of the CTST energetics. Indeed, the model will likely evolve as it is tested against an ever increasing matrix of QD-type, surface-modification and experimental conditions, as well as new PL phenomena. For example, evidence for different modes of blinking suggests A-type and B-type can coexist in QDs with their contributions weakly dependent on shell-thickness. Our results align closely with an A-type blinking mechanism, since grey-state emission levels observed in our experiments compare well with those reported elsewhere, in which PL lifetimes are found strongly correlated with PL intensity. However, the model is also consistent with B-type blinking where the exciton-hole equilibrium is shifted strongly toward the QD surface, by deep trapping at a dangling bond, for example. ${ }^{69}$ In this case, the dark, off-sate of the QD is associated with capture of hot electrons at the hole and the consequent inhibition of efficient band-edge emission. ${ }^{34}$ Ultimately, the CTST description advanced here offers a simple, but physically insightful basis for the interpretation of PL phenomena in QDs and similar photoactive nanomaterials, as well as the rational control of exciton dynamics and emission through material design and synthesis.

\section{Experimental}

\section{Materials and sample preparation}

All reported PI experiments used CdSe-ZnS QDs (Lumidot 590, Sigma-Aldrich, UK) diluted in toluene (TOL, Analytical Grade,
Fisher Scientific, UK). All chemicals were sourced from SigmaAldrich unless stated otherwise. QDs were incorporated in the supporting dielectric medium by diluting in a QD/polymer/ solvent mix or depositing the QD/TOL solution on a pre-made polymer support. Thin-film polymer supports were prepared by spin-coating $(3000 \mathrm{rpm})$ solutions on a coverslip $(22 \times 40$, \# 1.5, Menzel Glaser, EU) and allowing solvent evaporation for over $30 \mathrm{~min}$. Coverslips were flame-cleaned and ozonated for 30-60 min (PSD Series, Novascan, USA) to remove residual fluorescence prior to QD deposition and QD concentrations were adjusted to achieve surface densities of approximately $0.01 \mathrm{QD} \mu \mathrm{m}^{-2}$ to ensure good spatial separation of single QDs.

Support medium of $p$-terphenyl (pT, >98.5\% HPLC) was prepared at $3 \mathrm{mg} \mathrm{ml}^{-1}$ in TOL with QDs spin coated on top of the host-film. For polystyrene (PS, MW 192 000) $100 \mathrm{mg}$ of PS was dissolved in $6 \mathrm{~mL}$ of TOL and the QDs diluted into the PS/ TOL mix before spin-coating. Poly( $N$-vinylpyrrolidone) (PVP, MW 40000 ) was prepared from $100 \mathrm{mg}$ of PVP dissolved in $6 \mathrm{ml}$ of ethanol absolute (VWR Chemicals, UK) and QDs spincoated from TOL onto the polymer-film. Polyvinylalcohol (PVA, MW 130000 ) was prepared as a $3 \mathrm{mg} \mathrm{mL}^{-1}$ solution in deionised water, spin coated onto the coverslip and residual water left to evaporate until dry, with QDs deposited on top of the polymer film. For glass $\left(\mathrm{SiO}_{x}\right)$, QDs were spin-coated directly onto the pre-cleaned coverslip. Prepared coverslips were optically coupled to the objective lens of an inverted microscope via immersion oil (Olympus, Type F, $n=1.581$ ). For "grey"-state investigations CdSe-ZnS QDs (EviDot, birch yellow, em: $580 \mathrm{~nm}$, Evident Tech. Inc., USA) were diluted in the stock solvent TOL and spin-coated on a PVA support as above. Studies of PE and PL decay were performed on ZnS capped CdSe QDs (EviDot, fort orange, em: $600 \mathrm{~nm}$ ), spin coated directly on cover glass, while control, core-only CdSe QDs were synthesised from cadmium-oleate (CdOA) and trioctylphosphine-selenide (TOP-Se) precursors using a standard high temperature $\left(250^{\circ} \mathrm{C}\right.$ ) injection procedure (note S9 in ESI $\dagger$ ). ${ }^{70}$

\section{Image acquisition}

QD imaging was performed on a modified, inverted microscope (TE2000-U, Nikon UK) using objective-type TIRF. A $473 \mathrm{~nm}$ CW laser (Scitec Instruments, UK) operating was coupled through the objective lens (Plan Apo, 60×, NA 1.45) to the sample via a dichroic beamsplitter (BS, Di01-R488-561, Semrock, USA). The excitation beam was made near-collimated at the sample using a $200 \mathrm{~mm}$ plano-convex TIRF lens focused off-axis at the back focal-plane of the objective to achieve TIR. For PI experiments the TIRF lens was adjusted to obtain an excitation footprint of $50 \mu \mathrm{m}$ in diameter with an intensity of $45 \mathrm{~W} \mathrm{~cm}^{-2}$, accounting for near-field enhancement. For "grey"state studies the intensity was raised to $80 \mathrm{~W} \mathrm{~cm}^{-2}$, while PE was typically observed at power densities upward of $0.5 \mathrm{~kW}$ $\mathrm{cm}^{-2}$. PL collected by the objective lens was separated from laser scatter at the dichroic, passed through a bandpass filter (BP, Semrock, Brightline 609/54) and detected on a watercooled ICCD camera (Princeton Instruments, PI-Max 512 GenIII). Image-stacks with 12 bit grey-scale digitisation, were 
recorded with $80 \mathrm{~ms}$ integration per frame (12.5 fps) for up to 20 min providing typically 15000 frames per movie for PI experiments. For "grey"-state investigations, a $50 \times 50$ pixel read-out region of the ICCD was used to reduce exposure times to $25 \mathrm{~ms}$ (40 fps). The microscope sample stage and focus drive (Proscan II, Prior Scientific, UK) and camera were controlled using open-source, image acquisition and processing packages, $\mu$ Manager and ImageJ. ${ }^{71}$ Focus-drift over extended acquisition times was eliminated using an active feedback loop that exploits the linear-relationship between the lateral displacement of the back-reflected TIR beam, imaged on an external camera (DCC1645C, Thorlabs, UK) and drift of the objective lens from focus. A motorized focus drive (PS3H122, Prior Scientific, UK) was controlled to maintain focus using a macro developed in ImageJ. For purposes of simulation we measured an overall detection efficiency of $1 \%$ including optical collection, quantum efficiency and analogue-to-digital (ADU) grey-level conversion, a camera offset of 50 cts and excess noise factor of 1.6 for the ICCD. ${ }^{72}$

\section{Image processing and data analysis}

QD image stacks were analysed and single QDs identified by their diffraction-limited intensity profile (FWHM $\sim 200 \mathrm{~nm}$ ) and binary-like blinking in the intensity trajectory. For each PL trajectory accepted for analysis, a threshold corresponding to $2 \sigma$, from the dark-state mean and close to the minimum in the intensity histogram of bright and dark populations, was used to distinguish the radiative on-state from the dark-off state. The on- and off-times corresponding to periods in the trajectory where PL remained above and below threshold respectively were extracted and the PDD for each calculated as $P\left(t_{i}\right)=$ $2 N_{i} /\left[\left(t_{i+1}-t_{i}\right)+\left(t_{i}-t_{i-1}\right)\right]$, where $N_{i}$ is the number of occurrences of a given on/off event of duration $t_{i}$ and $t_{i+1}$ and $t_{i-1}$ are the durations of proceeding and preceding events respectively. ${ }^{32}$ The PDDs extracted were fitted with the TPL, $P(t)=$ $A t^{-\alpha} e^{-t / \tau_{\mathrm{c}}}$, by varying parameters $A, \alpha$ and $\tau_{\mathrm{c}}$ using a LevenbergMarquardt algorithm for non-linear least-squares minimisation (Origin 8). The distribution of TPL parameters, $\alpha_{\text {on }}, \alpha_{\text {off }}$ and $\tau_{\mathrm{c}}(\mathrm{on})$ were derived from the PL intensity trajectories of over 25 QDs per (dielectric) sample, each recorded for typically 20-30 min and covering over 1000-3000 on/off switching events.

\section{Stochastic simulation methods}

QD PL intensity trajectory simulations were performed using a stochastic simulation algorithm. ${ }^{73}$ The algorithm samples both the time a QD spends in a given state and the transition that occurs after this time in a probabilistic manner and is particularly suited to the highly distributed kinetics of QD blinking, where on- and off-states can persist for long periods $>10^{2} \mathrm{~s}$. The time, $\tau$, spent by the QD in any given state and the transition $m$ that occurs after this time are selected from the probability density distributions, $r_{0} e^{-r_{0} \tau}$ and $r_{\mathrm{m}} / r_{0}$, respectively, where $r_{0}=\sum r_{i}$ is the sum of transition rates out of the current state and $r_{i}=k_{\mathrm{i}} f_{x}$, for each rate constant, $k_{\mathrm{i}}$ and the state filling fraction, $f_{\mathrm{x}}$. For a QD in a given state at time $t_{0}$, the algorithm proceeds by first selecting the time step to the next transition using the inverse transform $\tau=-\ln \left(u_{1}\right) / r_{0}$ and the transition that follows according to the condition $\sum_{i=1}^{m} r_{i}<r_{0} u_{2} \leq \sum_{i=1}^{m+1} r_{i}$, where $u_{1}$ and $u_{2}$, are uniform random numbers in the interval $[0,1]$. The microscopic simulation time $t$ is incremented by $\tau$ and properties of the QD updated to the new state defined by $m$ before the process is repeated. Events are integrated in the time interval $t_{\text {int }}$, while $t+\tau \leq t_{0}+t_{\text {int }}$, after which the macroscopic observation time $t_{0}$ is incremented by $t_{\text {int }}$ and the process repeated.

\section{Acknowledgements}

This research was partly carried out and funded under the Royal Society University Research Fellowship Award of M.A.O. The work of A.F. was funded through a Doctoral Training Assistant (DTA) awarded by the School of Life Sciences, University of Sussex, UK.

\section{References}

1 J. A. Smyder and T. D. Krauss, Mater. Today, 2011, 14, 382-387. 2 L. E. Brus, J. Chem. Phys., 1983, 79, 5566-5571.

3 M. Nirmal, B. O. Dabbousi, M. G. Bawendi, J. J. Macklin, J. K. Trautman, T. D. Harris and L. E. Brus, Nature, 1996, 383, 802-804.

4 M. Kuno, D. P. Fromm, H. F. Hamann, A. Gallagher and D. J. Nesbitt, J. Chem. Phys., 2000, 112, 3117-3120.

5 K. T. Shimizu, R. G. Neuhauser, C. A. Leatherdale, S. A. Empedocles, W. K. Woo and M. G. Bawendi, Phys. Rev. B: Condens. Matter, 2001, 63, 205316.

6 R. Schmidt, C. Krasselt, C. Gohler and C. von Borczyskowski, ACS Nano, 2014, 8, 3506-3521.

7 S. R. Cordero, P. J. Carson, R. A. Estabrook, G. F. Strouse and S. K. Buratto, J. Phys. Chem. B, 2000, 104, 12137-12142.

8 M. Jones, J. Nedeljkovic, R. J. Ellingson, A. J. Nozik and G. Rumbles, J. Phys. Chem. B, 2003, 107, 11346-11352.

9 T. Uematsu, J. Kimura and Y. Yamaguchi, Nanotechnology, 2004, 15, 822-827.

10 C. Carrillo-Carrion, S. Cardenas, B. M. Simonet and M. Valcarcel, Chem. Commun., 2009, 5214-5226.

11 W. G. J. H. M. van Sark, P. L. T. M. Frederix, A. A. Bol, H. C. Gerritsen and A. Meijerink, ChemPhysChem, 2002, 3, 871-879.

12 Y. Zhang, J. He, P. N. Wang, J. Y. Chen, Z. J. Lu, D. R. Lu, J. Guo, C. C. Wang and W. L. Yang, J. Am. Chem. Soc., 2006, 128, 13396-13401.

13 S. F. Lee and M. A. Osborne, J. Am. Chem. Soc., 2007, 129, 8936-8937.

14 H. P. Chen, H. W. Gai and E. S. Yeung, Chem. Commun., 2009, 1676-1678.

15 T. D. Krauss and L. E. Brus, Phys. Rev. Lett., 1999, 83, 48404843. 
16 K. Zhang, H. Y. Chang, A. H. Fu, A. P. Alivisatos and H. Yang, Nano Lett., 2006, 6, 843-847.

17 P. Spinicelli, S. Buil, X. Quelin, B. Mahler, B. Dubertret and J. P. Hermier, Phys. Rev. Lett., 2009, 102, 136801.

18 D. E. Gomez, J. van Embden, P. Mulvaney, M. J. Fernee and H. Rubinsztein-Dunlop, ACS Nano, 2009, 3, 2281-2287.

19 A. L. Efros and M. Rosen, Phys. Rev. Lett., 1997, 78, 11101113.

20 R. Verberk, A. M. van Oijen and M. Orrit, Phys. Rev. B: Condens. Matter, 2002, 66, 233202.

21 J. Tang and R. A. Marcus, J. Chem. Phys., 2005, 123, 054704.

22 P. A. Frantsuzov and R. A. Marcus, Phys. Rev. B: Condens. Matter, 2005, 72, 155321.

23 B. Chon, S. J. Lim, W. Kim, J. Seo, H. Kang, T. Joo, J. Hwang and S. K. Shin, Phys. Chem. Chem. Phys., 2010, 12, 9312-9319.

24 Z. Y. Zhu and R. A. Marcus, Phys. Chem. Chem. Phys., 2014, 16, 25694-25700.

25 K. L. Knappenberger, D. B. Wong, Y. E. Romanyuk and S. R. Leone, Nano Lett., 2007, 7, 3869-3874.

26 C. H. Crouch, R. Mohr, T. Emmons, S. Y. Wang and M. Drndic, J. Phys. Chem. C, 2009, 113, 12059-12066.

27 K. Goushi, T. Yamada and A. Otomo, J. Phys. Chem. C, 2009, 113, 20161-20168.

28 K. T. Early and D. J. Nesbitt, Nano Lett., 2013, 13, 4844-4849.

29 J. Vela, H. Htoon, Y. F. Chen, Y. S. Park, Y. Ghosh, P. M. Goodwin, J. H. Werner, N. P. Wells, J. L. Casson and J. A. Hollingsworth, J. Biophotonics, 2010, 3, 706-717.

30 A. Issac, C. von Borczyskowski and F. Cichos, Phys. Rev. B: Condens. Matter, 2005, 71, 161302.

31 A. Issac, C. Krasselt, F. Cichos and C. von Borczyskowski, ChemPhysChem, 2012, 13, 3223-3230.

32 J. J. Peterson and D. J. Nesbitt, Nano Lett., 2009, 9, 338-345.

33 M. Kuno, D. P. Fromm, S. T. Johnson, A. Gallagher and D. J. Nesbitt, Phys. Rev. B: Condens. Matter, 2003, 67, 12304.

34 C. Galland, Y. Ghosh, A. Steinbruck, M. Sykora, J. A. Hollingsworth, V. I. Klimov and H. Htoon, Nature, 2011, 479, 203-207.

35 S. Maenosono, Chem. Phys. Lett., 2003, 376, 666-670.

36 S. J. Park, S. Link, W. L. Miller, A. Gesquiere and P. F. Barbara, Chem. Phys., 2007, 341, 169-174.

37 A. Javier and G. F. Strouse, Chem. Phys. Lett., 2004, 391, 60-63.

38 K. Pechstedt, T. Whittle, J. Baumberg and T. Melvin, J. Phys. Chem. C, 2010, 114, 12069-12077.

39 L. Manna, E. C. Scher, L. S. Li and A. P. Alivisatos, J. Am. Chem. Soc., 2002, 124, 7136-7145.

40 J. I. Kim, J. Kim, J. Lee, D. R. Jung, H. Kim, H. Choi, S. Lee, S. Byun, S. Kang and B. Park, Nanoscale Res. Lett., 2012, 7, 1-7.

41 K. Kohary and G. A. Gibson, Phys. Status Solidi C, 2011, 8, 9.

42 S. I. Yamashita, M. Hamada, S. Nakanishi, H. Saito, Y. Nosaka, S. I. Wakida and V. Biju, Angew. Chem., Int. Ed., 2015, 54, 3892-3896.

43 K. T. Early, K. D. McCarthy, N. I. Hammer, M. Y. Odoi, R. Tangirala, T. Emrick and M. D. Barnes, Nanotechnology, 2007, 18, 424027.
44 C. Krasselt, J. Schuster and C. von Borczyskowski, Phys. Chem. Chem. Phys., 2011, 13, 17084-17092.

45 Y. Shirasaki, G. J. Supran, M. G. Bawendi and V. Bulovic, Nat. Photonics, 2013, 7, 13-23.

46 Y. Wang, G. Fruhwirth, E. Cai, T. Ng and P. R. Selvin, Nano Lett., 2013, 13, 5233-5241.

47 A. Y. Nazzal, L. H. Qu, X. G. Peng and M. Xiao, Nano Lett., 2003, 3, 819-822.

48 J. W. M. Chon, P. Zijlstra, M. Gu, J. van Embden and P. Mulvaney, Appl. Phys. Lett., 2004, 85, 5514-5516.

49 F. S. Dainton, M. J. Pilling and S. A. Rice, J. Chem. Soc., Faraday Trans. 2, 1975, 71, 1311-1321.

50 K. H. Gundlach and J. G. Simmons, Thin Solid Films, 1969, 4, 61-79.

51 L. W. Wang and A. Zunger, Phys. Rev. B: Condens. Matter, 1996, 53, 9579-9582.

52 J. C. M. Garnett, Philos. Trans. R. Soc. London, Ser. A, 1904, 203, 385-420.

53 L. Banyai, P. Gilliot, Y. Z. Hu and S. W. Koch, Phys. Rev. B: Condens. Matter, 1992, 45, 14136-14142.

54 C. W. Bunn, Nature, 1948, 161, 929-930.

55 G. W. Bak, J. Phys. C: Solid State Phys., 1987, 20, 1129-1136.

56 J. F. Verwey, E. A. Amerasekera and J. Bisschop, Rep. Prog. Phys., 1990, 53, 1297-1331.

57 L. E. Brus, J. Chem. Phys., 1984, 80, 4403-4409.

58 M. Shim and P. Guyot-Sionnest, J. Chem. Phys., 1999, 111, 6955-6964.

59 B. S. Kim, M. A. Islam, L. E. Brus and I. P. Herman, J. Appl. Phys., 2001, 89, 8127-8140.

60 W. W. Yu, L. H. Qu, W. Z. Guo and X. G. Peng, Chem. Mater., 2003, 15, 2854-2860.

61 C. D. Donega and R. Koole, J. Phys. Chem. C, 2009, 113, 6511-6520.

62 V. I. Klimov, A. A. Mikhailovsky, D. W. McBranch, C. A. Leatherdale and M. G. Bawendi, Science, 2000, 287, 1011-1013.

63 M. A. Marcus, L. E. Brus, C. Murray, M. G. Bawendi, A. Prasad and A. P. Alivisatos, Nanostruct. Mater., 1992, 1, 323-335.

64 J. B. Sambur and B. A. Parkinson, J. Am. Chem. Soc., 2010, 132, 2130-2131.

65 C. H. Crouch, O. Sauter, X. H. Wu, R. Purcell, C. Querner, M. Drndic and M. Pelton, Nano Lett., 2010, 10, 1692-1698.

66 A. Makhal, H. D. Yan, P. Lemmens and S. K. Pal, J. Phys. Chem. C, 2010, 114, 627-632.

67 J. E. B. Katari, V. L. Colvin and A. P. Alivisatos, J. Phys. Chem., 1994, 98, 4109-4117.

68 M. A. Osborne and S. F. Lee, ACS Nano, 2011, 5, 8295-8304.

69 N. P. Brawand, M. Voros and G. Galli, Nanoscale, 2015, 7, 3737-3744.

70 R. G. Xie, U. Kolb, J. X. Li, T. Basche and A. Mews, J. Am. Chem. Soc., 2005, 127, 7480-7488.

71 C. A. Schneider, W. S. Rasband and K. W. Eliceiri, Nat. Methods, 2012, 9, 671-675.

72 R. L. Boulineau and M. A. Osborne, Chem. Commun., 2013, 49, 5559-5561.

73 D. T. Gillespie, J. Phys. Chem., 1977, 81, 2340-2361. 